\title{
EDUCATIONAL PLANNING FOR SYSTEMS- ORIENTED DESIGN: APPLYING SYSTEMIC RELATIONSHIPS TO META-MAPPING OF GIGA MAPS
}

\author{
Linda BLAASVAR ${ }^{1}$ and Birger SEVALDSON ${ }^{2}$ \\ ${ }^{1}$ Norwegian Labour and Welfare Administration \\ ${ }^{2}$ Oslo School of Architecture and Design
}

\begin{abstract}
We have sought to develop pedagogy in systems-oriented design (SOD; Sevaldson, Nordic Design Research 2011: Making Design Matter, 2011), with the objective of helping design students become well versed with systems. SOD requires an understanding of the relationships between elements in a system. This article explores the SOD method of GIGA mapping developed at The Oslo School of Architecture and Design (AHO), Norway, in relation to design literacy and educational planning at bachelor's and master's levels. GIGA mapping is a method that is based on systems thinking and design practice and uses visualisation as the primary technique to explore exceedingly complex situations, structures, and systems. Our comprehensive collection of GIGA maps collected from AHO's courses since 2010 shows a great variation in qualities such as communication, complexity, and richness. By analysing more than 100 GIGA maps, we were able to see patterns emerge from the way students created and structured the content. We categorised the GIGA maps into six different levels of complexity, using Bloom's structure of the knowledge dimension of the revised taxonomy (Bloom and Krathwohl, Taxonomy of Educational Objectives, Volume 2: Affective Domain, 1971). We called these six levels of complexity the "GIGA mapping ladder." The ladder describes progressively more complex stages of introducing the vast knowledge, methods, and skills in GIGA mapping during the course of SOD education. We present this pedagogical model as a way of helping students learn and advance in a complex topic.
\end{abstract}

Keywords: Educational planning, design process, systems-oriented design, systemic relationships, GIGA mapping, design research.

\section{GIGA MAPPING IN THE FIELD OF SYSTEMIC DESIGN AND SYSTEMS- ORIENTED DESIGN}

Systems can be seen as the "relationship between structure and behaviour" [3], and they represent complex networks of relations in our society, environment, and so forth. The design methods through which you would explore society and environment are, therefore, linked to complexity and, in extension systems, to theory and design. Designers need methods to handle such complexity. This is how GIGA maps have emerged. Systems-oriented design (SOD) seeks to train designers to become better at dealing with complex issues. As design is moving into new fields of services and public, as well as private, organisations, the need to understand complex systems and design for them is increasing. Designers are potentially in a good position to have a holistic overview to manage complex tasks, but their systemic insight is limited despite earlier theorising of systems approaches in design [4, 5]. Designers need to be aware of how their actions can have unintended effects and counterintuitive kickbacks from systems change. SOD lies within the broader field of systemic design [11]. It is systems thinking tailored for practicing designers. Today, research refers to three main conceptual frameworks: (1) design thinking and design practice; (2) visual thinking and visual practice; and (3) systems thinking and systems practice [1]. This development was oriented toward design practice rather than toward importing models, methods, and approaches from other systems fields, such as systems dynamics or cybernetics. Although these and many others, such as soft systems methodology, have been important to SOD, the 
main intention was to develop a designerly way of dealing with complex systems. Methods to analyse such complexity and to understand relations in a system through systems modelling was experimenting with different time horizons, understanding cause and effect relationships, and relationships between reinforcing and balancing feedback loops [3]. The main output has been the concept of GIGA mapping. GIGA mapping is very extensive mapping that crosses perceived boundaries and scales. The intention is manifold, but we can briefly mention; to build a deep understanding of the system at hand and its environment and wider landscape; to initiate a very rapid learning process; to tease out the "unknown unknowns"; to serve as a dialogic tool across silos and disciplines; to engage stakeholders and nonstakeholders; to cater to sustainability, life cycles, and circular economy issues; to serve deep creative processes; and to memorise large amounts of information and insights. GIGA mapping is a powerful tool for visualising design research, as well as for inquiry in a professional context [6]. Typically, a GIGA map would be a rich visualisation - a construct of an image of a system developed as a design artefact over several iterations. The technique of GIGA mapping is simple and intuitive at the outset, but it has a vast potential to implement ever more advanced techniques and address increasingly difficult challenges. It is more skill-based than method-based and mastering the more complex applications requires training. Therefore, a stepwise progression in the acquirement of GIGA mapping skills would be beneficial. One of the difficulties that faces a practitioner of GIGA mapping after the first easy steps are mastered is to understand systemic relations better. In GIGA mapping, attention needs to be moved from the entities in a system to the relations. To do this, we have developed a library to describe a multitude of different types of relations that emerge from mapping. The Library of Systemic Relations is only described briefly in this article [7].

\section{METHOD}

We have taught SOD in bachelor's studios in addition to SOD in master's courses. Since the first bachelor's studio during the spring semester of 2015, we have witnessed the need to differentiate systems concepts and SOD methods to suit a bachelor's or master's student. Through this article, we seek to explore pedagogic methods to teach systemic relationships informed by the emerging diversity of GIGA maps developed in our courses, to develop SOD pedagogy. Using the GIGA map samples [12], we analysed this growing library for emerging patterns in GIGA mapping, in the context of planning for SOD pedagogy. The GIGA maps gathered through teaching SOD to design students makes the total empirical data for this research. The sample collection of GIGA maps is an archive of student work from The Oslo School of Architecture and Design (AHO, Norway), Oslo Met (Norway), OCAD University (Canada), and our extended professional network. We drew on the 12 categories presented in "Gigamapping: Visualisation for Complexity and Systems Thinking in Design" [1]. These categories were later updated and further described by Skjelten [13]: (1) Timelines, (2) Network, (3) Spatial, and (4) Exploratory; additional typologies by Paulsen et. al. [14].: (1) Context, (2) Sequential, (3) Relations, and (4) Exploratory.

\subsection{What did we do?}

GIGA maps form a system of practice or praxeology in their own right, so to perform the analysis and investigation, we used GIGA mapping as a form of meta-mapping. We analysed a hundred GIGA maps; and six different levels of complexity emerged related to the method of GIGA mapping. We classified the different maps into typologies that represented a sort of similarity through manual mapping. The explanations that describe the specifics of each typology were given names. To discuss these typologies up against pedagogics, we compared the complexity of the GIGA maps with Bloom's taxonomy-more specifically, the structure of the knowledge dimension of the revised taxonomy [2]. We called these six steps of increasing complexity the "GIGA mapping ladder."

The second important focus of the study was to match a selection of systemic relations with the six types of GIGA maps, as SOD requires understanding of the relationships between elements in a system. We started to look for the patterns of use and design and the build-up of maps. It was clear from the beginning that this should not be carved in stone and that a hard categorisation would not only be useless but also produce a false sense of order and certainty. Therefore, we looked at this as a soft sorting of the patterns related to the use and design of maps. 


\section{BLOOM'S TAXONOMY}

SOD is hard to grasp in that it is non-existent as a visual or concrete condition [3]. Our experience of teaching SOD for a decade has been that learning about and attaining skills in SOD is often a long and frustrating maturing process. Accordingly, the pedagogical layout, strategy, or perspective is of importance in order to both motivate and help students to grasp the content of the methodology and apply it in design work. As Bateson pointed out, learning as a process leads to change, and he further suggested that "... we should begin the ordering of our ideas about 'learning' at the very simplest level" [9]. The introduction of systems theory in design education certainly leads to a change in the student's perspective of design and future practice. However, the complexity that SOD represents makes it difficult to establish what represents the simplest level of learning up against performance and creation. To explore levels of complexity in attaining knowledge and skills in SOD, we turned to Bloom et al. and their research on pedagogics and steps of learning. Bloom's taxonomy describes six general levels within the cognitive domain that relate to the stages of learning from simple to complex and from concrete to abstract - that is, the taxonomy also suggests a sequence of various learning outcomes for students within these parameters. Further, it suggests how the attainment of learning outcomes may build on each other or how they make the next level of learning possible or more giving. A teacher can, thus, make use of the taxonomy to plan and describe the potential learning outcomes produced by specific sequences in the educational planning. Hence, Bloom regarded the taxonomy as a tool to analyse, plan, and measure the effect of education. In general terms, it may serve as (1) a common language for learning goals (to facilitate communication across persons, subject matter, and grade levels); (2) a basis for determining a particular course or curriculum/broad educational goals (national/state/local standards); (3) a means for determining congruence of educational objectives, activities, assessments in a unit, course, or curriculum; and (4) a panorama of the range of educational possibility - any particular educational course or curriculum could be contrasted [10].

Table 1. the six steps in the GIGA mapping ladder with the "original" and "revised" taxonomy

\begin{tabular}{|c|c|c|c|}
\hline & Original taxonomy (1956) & Revised taxonomy (2001) & GIGA mapping ladder (2019) \\
\hline 1 & Knowledge & Remembering & Categorising \\
\hline 2 & Comprehension & Understanding & Systemic relationships \\
\hline 3 & Application & Applying & Contexts \\
\hline 4 & Analysis & Analysing & Synthesis \\
\hline 5 & Synthesis & Evaluating & Effects (evaluate) \\
\hline 6 & Evaluation & Creating & Creating \\
\hline
\end{tabular}

\section{IMPLICATIONS FOR DESIGN}

The GIGA map is a tool for design as inquiry and for knowledge production. It is a tool for research through design. GIGA mapping initiates very rapid learning processes, as mentioned previously, and supports and strengthens learning through the design process. The design sketch explores objects and services through visual thinking [11], and reflection in action [8], whereas the GIGA map helps to do the same with abstract structures, systems, and relations. Systemic relations concern the analysis of the ties and nodes that occur and influence structures and systems, all of which can be visualised in a GIGA map. However, the GIGA mapping is a general process and research tool. GIGA mapping is also introduced to first year students in design at AHO since 2017. This introduction is a 2-day seminar to introduce students in all design disciplines to a tool for a systemic approach to tackle design challenges. We hope The Ladder will function as a teaching support that will cater for the students' elevation and ability to become experts in understanding exceedingly complex systems. Cognitive development is limited to a certain range as Zone of Proximal Development [15]. The ladder presents a range of skills that can be developed building on the prior skills and knowledge foundation for the student to succeed.

\section{DISCUSSION}

Bloom's taxonomy creates and sustains a communication and planning platform for education internally and across institutions. Therefore, we used this taxonomy as a means to analyse and reflect on how to teach SOD, and in what sequence, the various associated methods should be introduced, which by working together produce different learning outcomes. We made a draft of the six steps of the GIGA mapping ladder as a guideline to impart appropriate levels of learning at each step and, at the same time, 
to focus on the curricular objectives (see Table 1). One of the most frequent uses of the original taxonomy is to classify curricular objectives and test items in order to show the breadth, or lack of breadth, of the objectives and items across the spectrum of categories. These analyses almost always lay a heavy emphasis on objectives requiring only recognition or recall of information. According to Bloom, these objectives belong to category 1 "knowledge." Objectives that involve the understanding and application of knowledge would be classified into categories 2 "comprehension" to 6 "evaluation." The span of learning that these five categories, from $2-6$ embraces, includes the most important goals in higher education - especially where there is emphasis on creation on the basis of skills and theory. Thus, we strived for our students to achieve a level of expertise where all SOD tools and techniques can be used effortlessly along with a conscious and critical view toward how they are applied and adjusted in relation to their influence on the potential outcome. Analyses have repeatedly provided a basis for moving curricula and tests toward objectives that are classified in exceedingly complex categories. In our SOD teaching, we aimed at creating an education sequence using the reverse engineering method; that is, we tried to adjust our pedagogy to bridge the gap between beginner's and master's level, as well as to make GIGA mapping more accessible and self-explanatory from the very beginning [10]. Krathwohl referred to an interesting process of reclassifying the different categories in order to make them more accurate in relation to how educators understand and describe the different terms in the taxonomy-for example, “... when teachers say that they want the student to really understand, they mean anything from Comprehension to Synthesis" [10]. Therefore, the category of "knowledge" was renamed to Remember to rule out the stages of learning that belong to the higher levels of the taxonomy, such as Synthesis and Evaluation [10]. As Krathwohl suggested, "the revision is a hierarchy in the sense that the six major categories of the Cognitive Process dimension are believed to differ in their complexity, with remember being less complex than understand" [10]. This revision reflects the educational planning for SOD in a practical manner as SOD students are in fact introduced to systems and complexity theory to be remembered, in addition to performing analysis and creating products and services in complex contexts. In this process, students climb the ladder from remembering systems and complexity theory to being able to work within complexity. That said, in all instances of the educational planning for SOD, we rely on students who have either completed their bachelor's studies or completed at least a couple of courses of their bachelor's studies, where they have attained skills in two- or threedimensional visualisation of contexts, elements, relationships, graphs, and so on. Krathwohl's revised taxonomy extracts knowledge as one primary dimension with four subdimensions and the cognitive process as another dimension with six categories. In addition to guiding a student to elevate through the six steps toward an increasingly independent use of the SOD tools and techniques to handle complex problem-solving, we can use the steps in this taxonomy to analyse the GIGA mapping ladder and support us in reflecting on the SOD curriculum and pedagogic developments, as we can map out the student hand-ins according to the steps. Subsequently, we can use this analysis to adjust and renew future SOD courses. This work is important for us to make a leap in our pedagogy as "the panorama of possibilities presented by the taxonomy table causes one to look at blank areas and reflect on missed teaching opportunities" [10]. All categories seem immediately suggestive, except the discussion board, which opens up for own thinking and reflection.

\subsection{A selection of types of Systemic Relations}

The Library of Systemic Relations consists of 30 variations [7]. We selected six types of relations with a pedagogic perspective in mind-(1) Semantic relations, (2) Social relations, (3) Actions (over time), (4) Structures and Physical relations, (5) Causal relations, and (6) Feedback loops. These types were selected after looking at the patterns of use of GIGA maps, and the usefulness to apply different relations to different types of maps, according to Bloom's taxonomy of learning, and to further allow the six steps of the GIGA mapping ladder to merge. The selection of relation $1-3$ was chosen to build upon students - existing knowledge from design education semantic relation and their experience in the world social relations and actions. Structures and physical relations may represent hard structures of systems, these are elements we can actually see and is therefore proposed as the next relation to be introduced in the ladder. The fifth relation in the ladder is casual relations and we ask students to look for cause and effect relationships in a low-level system dynamic type of GIGA map. The sixth relation is feedback loops where we ask students to further build on the system dynamic map, elevate towards a high-level GIGA map and add reinforcing - and balancing feedback loops. The high-level GIGA map will present and 
reflect on how systems work by visually describing for example the situation under investigation, connection to the surrounding landscapes, dependencies, relationships, implications, and so forth.

\section{THE GIGA MAPPING LADDER - A NEW PEDAGOGICAL TOOL}

There are several types of GIGA maps that enables students to understand systemic relationships. The synthesis from the GIGA map library and the selection of systemic relations creates the foundation for the six steps of increasing complexity in the GIGA mapping ladder. The model also includes the six steps of the revised taxonomy: (1) Remembering, (2) Understanding, (3) Applying, (4) Analysing, (5) Evaluating, and (6) Creating (see Table 2).

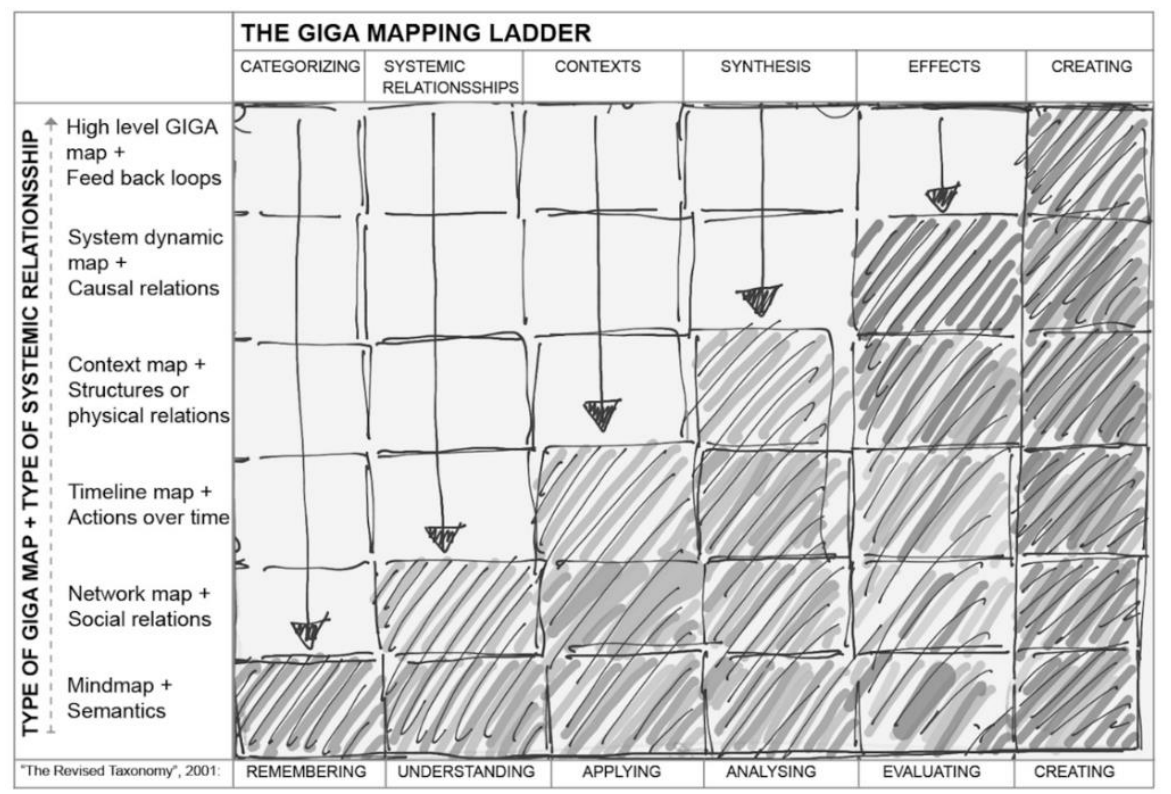

Table 2. The GIGA mapping ladder

In the first step of the ladder Categorising, the student will work with what is related and how items can be organised into different categories: (1) the type of map-mind map; (2) look for one type of relation - semantic relations; and (3) the first level of Bloom's revised taxonomy-remembering. In the second step of the ladder Systemic relationships, the student will work with social relationships and look for different kinds of networks and how they are connected. They will decide on a way to visually differentiate the various networks, find out how they are related, and look for complexity in networksit is the first step in connecting the clouds of information: (1) the type of map-network map; (2) systemic relation - social relations; and (3) level of taxonomy - understanding. In the third step of the ladder Contexts, the student will work with actions playing out over time, including experiments with different time horizons and applying the content of maps to various contexts: (1) the type of maptimeline map; (2) systemic relation - actions; and (3) level of taxonomy - applying. In the fourth step of the ladder Synthesis, the student will build on the previous map and dig deeper into the context and try to detect different structures in systems: (1) the type of map-context map; (2) systemic relationstructures or physical relations; and (3) level of taxonomy - analysing. In the fifth step of the ladder Effects, the student will start to work with mixed maps, blending, relating, and looking for cause-andeffect relationships: (1) the type of map-systems dynamics map; (2) systemic relation - causal relations; and (3) level of taxonomy - evaluating. In the sixth step of the ladder Creating, the student will continue to work with mixed maps, blending and relating different maps, practicing the skill of freestyling by intuition, connecting and relating seemingly disconnected entities, and experiment with different ways of representing data, visually representing positive and negative feedback loops, and looking for things that accumulate. The student will work with quantifiable relations: (1) the type of map-high-level GIGA map; (2) systemic relation-feedback loops; reinforcing / positive and balancing / negative feedback loops; and (3) level of taxonomy — creating. 


\section{CONCLUSIONS AND FURTHER WORK}

Until now SOD pedagogy has been organic in the sense that we ask the students to dive into complex matters and facilitate learning by responding to their actions. Teaching on entry-levels revealed the need for a more step-by-step and ordered pedagogy: The GIGA mapping ladder. The ladder will be tested with bachelor's and master's students where they will be invited to a codesign process to refine the ladder. We suggest further research about the results from such test runs. After these applications, we might be able to draw some conclusions and compare it to the more organic and open pedagogy we have practiced so far.

\section{REFERENCES}

[1] Sevaldson B. (ed.) GIGA-mapping: Visualisation for complexity and systems thinking in design. Nordic Design Research 2011: Making Design Matter, 2011 (Nordes, Helsinki).

[2] Bloom B.S. and Krathwohl D.R. Taxonomy of Educational Objectives, Volume 2: Affective Domain, 1971 (David McKay Company, New York).

[3] Meadows D.H. and Wright D. Thinking in Systems: A Primer, 2009 (Earthscan, London).

[4] Ackoff R.L. (ed.) On Passing Through 80. Russel L Ackoff and the Advent of Systems Thinking, 1999 (Villanova University, Villanova).

[5] Banathy B.H. Designing Social Systems in a Changing World, 1996 (Springer, New York).

[6] Sevaldson B. and Alex J.R. Relating systems thinking and design I: Practical advances in systemic design. FormAkademisk, 2014, 7(3), pp. 1-5.

[7] Sevaldson B. Library of systemic relations. Available: http://www.systemsorienteddesign.net/index.php/tools/types-of-systemic-relations [Accessed 2019, 05,26], (2012), 26 October.

[8] Schön D.A. The Reflective Practitioner: How Professionals Think in Action, 1995 (Arena, Aldershot).

[9] Bateson G. Steps to an Ecology of Mind, 2000 (University of Chicago Press, Chicago).

[10] Krathwohl D.R. A revision of Bloom's taxonomy: An overview. Theory Into Practice, 2002, 41(4), pp. 212-218.

[11] Arnheim R. Visual thinking. London: Faber and Faber; 1969.

[12] Sevaldson B. GIGA-maps Samples 2012 [Available from: https://www.systemsorienteddesign.net/index.php/giga-mapping/giga-mapping-samples.

[13] Skjelten E.B. Complexity \& other beasts : a guide to mapping workshops. Oslo: The Oslo School of Architecture and Design; 2014.

[14] Paulsen A, Romm J, editors. Professional applications of Systems Oriented Design: Developments in practice. RSD2 Relating Systems Thinking and Design 2013.

[15] Vygotskij LS, Cole M, John-Steiner V, Scribner S, Souberman E. Mind in society: the development of higher psychological processes. Cambridge, Mass.: Harvard University Press; 1978. 159 s. : ill p. 